\title{
The Design and Optimization of Combined Rogowski Coil Based on Printed Circuit Board
}

\author{
Pongchit Saetang ${ }^{1}$ and Amnart Suksii ${ }^{1,2}$ \\ ${ }^{1}$ Department of Electrical Engineering, Faculty of Engineering, Khon Kaen University, Khon Kaen, 40002, Thailand, Tel. 043-347-031, \\ Fax. 043-347-032 \\ ${ }^{2}$ Center for Alternative Energy Research and Development, Department of Mechanical Engineering, Faculty of Engineering, \\ Khon Kaen University, Khon Kaen, 40002, Thailand, Tel. 0804614416
}

\begin{abstract}
Rogowski coil has been widely used for measurement of small magnitude with fast rise time signal such as partial discharge (PD), but Rogowski coil based on Printed circuit board (PCB Rogowski) has limitation for this application. This paper presents the prototype of Combined Rogowski coil that can overcome the drawback of PCB Rogowski coil. Experimental results showed that the proposed coil was able to measure a simulated of PD signal and its output signal indicated that the coil is suitable for a detection of small magnitude signal when compare with the other coils. The proposed Combined Rogowski coil in this research has upper frequency $\left(f_{h}\right)$ of $53.59 \mathrm{MHz}$ and mutual inductance $(M)$ of $0.417 \mu \mathrm{H}$, while PCB Rogowski coil has $f_{h}$ of $22.48 \mathrm{MHz}$ and $M$ of $0.026 \mu \mathrm{H}$. The oscillation of signal output that caused by non-uniform winding also has diminished. The electrical parameters that affected the performance of coil were controlled by the geometric variables and optimized by multi-objective genetic algorithm to achieve the optimum design of coil.
\end{abstract}

\section{Introduction}

Rogowski coil is a toroidal coil wound on a nonmagnetic core form. The coil has been widely used for the detection and measurement of electric currents in many applications [1]. Rogowski coil has many advantages such as linearity, has no effects from saturations, galvanic isolation and low cost. However, traditional coil is sometime hard to achieve on uniform winding that can cause errors to output measurement [2]. PCB Rogowski coil is designed by CAD methods, a perfect spacing is easy to achieve. Nevertheless, PCB Rogowski coil has a narrow bandwidth and very low sensitivity because inherent defect from its structure [3] meaning PCB Rogowski coil is not suitable for the detection of small and wide bandwidth signal such as partial discharge signal.

In this paper, a Combined Rogowski coil is proposed. The design coil was looking into an optimization of the bandwidth and sensitivity of the coil. It is sought by using genetic algorithm for multi-objective optimization technique.

\section{Rogowski coil principle}

Rogowski coil was developed by Rogowski and Steinhas in 1912. It has been made by placing the winding on a flexible or rigid toroidal core and then bending it round the conductor whose current is going to be measured.
Rogowski coil works by sensing the magnetic field in space around the conductor, Ampere's Law and Faraday's Law provide the relationship between current in the conductor $i_{p}(t)$ and output of Rogowski coil $V_{c}(t)$ as

$$
V_{c}(t)=-M \frac{d i_{p}(t)}{d t}
$$

where

$$
M=\frac{\mu_{0} N h}{2 \pi} \ln \frac{b}{a}
$$

Here $M$ is the mutual inductance between conductor and Rogowski coil, $\mu_{0}$ is the magnetic permeability of air $\left(4 \pi \times 10^{-7} \mathrm{H} / \mathrm{m}\right), a$ is inner radius, $b$ is outer radius, $h$ is height of core and $N$ is number of turns. In order to cancels external fields, the winding can be formed by returning the wire through the center of winding [4]. As in Fig. 1, shows the dimension of Rogowski coil. The behavior of Rogowski coil can be represented by the equivalent circuit of lumped parameter model [5] as shown in Fig. 2.

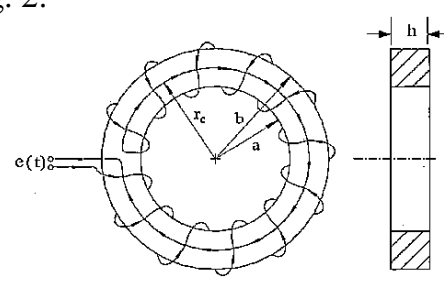

Figure 1. Rogowski coil. 


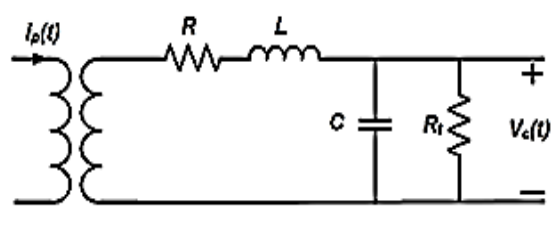

Figure 2. Lumped parameter of Rogowski coil.

Electrical parameters for rectangular cross-section of coil can be calculated as follows:

$$
\begin{gathered}
R=\frac{\rho l}{\pi\left(\frac{d}{2}\right)^{2}} \\
L=\frac{\mu_{0} N^{2} h}{2 \pi} \ln \frac{b}{a} \\
C_{l}=\left(4 \pi^{2} \varepsilon_{0} k \frac{b+a}{2}\right) /\left(\log \frac{b+a}{2} / \frac{b-a}{2}\right) \\
C_{t}=\pi \varepsilon_{0} k l / N \log \left(\frac{\pi(a+b)}{N d}+\sqrt{\pi(a+b)^{2} /(\mathrm{Nd}-1)}\right.
\end{gathered}
$$

where $R, L, C$ are the resistance, inductance and capacitance of coil, $\rho$ is electrical resistivity, $l$ is length of wire or length of imprint, $d$ is wire diameter, $\varepsilon_{0}$ is the electric permittivity of air $\left(8.854 \times 10^{-12} \mathrm{~F} / \mathrm{m}\right)$ and $k$ is relative permittivity.

The coil's capacitance $C$, composed of two capacitances in Rogowski coil. The first one is the turn to return capacitance of coil, $C_{l}$ can be calculated as acoaxial system [6] and the second one is the turn to turn capacitance of coil, $C_{t}$. The capacitance between turns would be a chain of series capacitors, $C_{t}$ which is smaller than $C_{l}$ and it can be neglected [7]. From the lumped parameters model, the transfer function $H(s)$ for this model is given by

$$
H(s)=\frac{V_{\text {out }}(s)}{I_{p}(s)}=\frac{\frac{M}{L C} s}{s^{2}+\left(\frac{L+R R_{t} C}{R_{t} L C}\right) s+\left(\frac{R+R_{t}}{R_{t} L C}\right)}
$$

where $R_{t}$ is damping resistor.

The variation of electrical parameters that affect the frequency response of the coil such as inductance and capacitance of coil are shown in Fig. 3,4.

When $L$ is increased by adding more number of turn or increase the height of core, a decrease of the sensitivity in the high frequency band is found. So, the inductance should be suitable so that it will result in high sensitivity while maintaining the sensitivity in the high frequency band. Increasing of $C$ will lead to the reduction of upper frequency response while maintaining sensitivity. The capacitance has to be kept as low as possible to avoid the drop of high frequency.

As for now, there are many kinds of PCB Rogowski coil, but most of them are made by replacing the windings by imprint of PCB then connected them into a loop, and the height of core is the thickness of baseboard. Since baseboard is very thin and it has high relative permittivity, resulting to the PCB Rogowski coil has low sensitivity and large capacitance. Therefore PCB Rogowski coil is not suitable for the high frequency application.

We propose a Combined Rogowski coil which is quite similar to the PCB Rogowski coil but the Combined Rogowski coil uses copper wire as a half loop to be connected to the baseboard that has the imprint as half loop for a complete coil. While PCB Rogowski coil uses only imprint on $\mathrm{PCB}$ as a loop. There are three advantages of this design. Firstly, the oscillation of output signal caused by reflection of electromagnetic wave due tonon-uniform winding is diminishing. Second, using a copper wire as a half loop makes the coil has low capacitance because core is an air instead of baseboard material which has a low value of permittivity. (As a result would improve the upper frequency of coil) Lastly, copper wire made coil's cross-section larger than PCB Rogowski coil, which is a mean of improving the sensitivity of the coil.

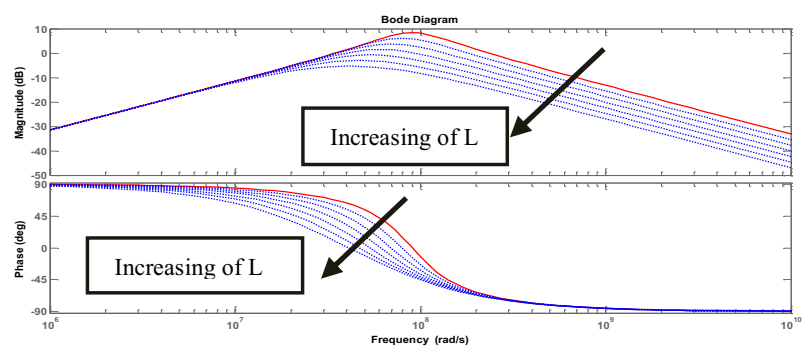

Figure 3. Inductance $L$ changes.

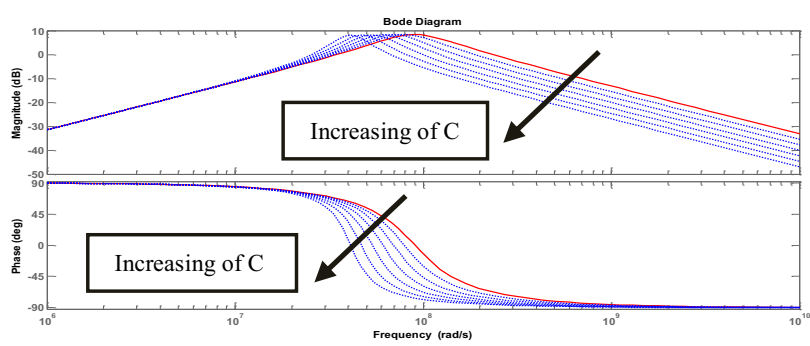

Figure 4. Capacitance $C$ changes.

\section{Experimental measurement}

Two Rogowski coils, PCB Rogowski coil (RC1), Combined Rogowski coil (RC2) have been constructed. The parameters of constructed coil are present in Table 1 and bode plot of two coils are shown in Fig.5. It can be proved that Combined Rogowski coil (RC2) has higher upper frequency and sensitivity than PCB Rogowski coil (RC1) when considering with the same size (inner, outer radius) and number of turns. Combined Rogowski coil may be more feasible for signal of small amplitude and fast rise time current signal measurement than PCB Rogowski coil.

The measuring were made using $\mathrm{RC} 1, \mathrm{RC} 2$ and nonuniform Rogowski coil are shown in Fig. 6, from left to right. These coil were clamped on XLPE cable to detect the signal from PD generator. The output of coils are measured by Tektronix TDS 1001B oscilloscope.

The model of PD generation is a needle-plate electrode configuration. It consisted of needle electrode 
which connects to high voltage and plate electrode as a return that connected to ground. The needle-plate gap was setup to $1 \mathrm{~mm}$. The current pulse of PD model and its frequency spectrum are shown in Fig. 7, Fig. 8.

Table 1. Parameters of constructed coils.

\begin{tabular}{|c|c|c|}
\hline \hline Parameters of coils & RC1 & RC2 \\
\hline Inner radius $a(m)$ & 0.03 & 0.03 \\
\hline Outer radius $b(m)$ & 0.09 & 0.09 \\
\hline Height of core $h(m)$ & 0.0032 & 0.05 \\
\hline Inductance $L(\mu H)$ & 1.0153 & 15.864 \\
\hline Mutual inductance $M(\mu H)$ & 0.02671 & 0.41747 \\
\hline Capacitance $C(p F)$ & 121.03 & 30.257 \\
\hline Resistance $R($ Ohm $)$ & 0.6695 & 0.3928 \\
\hline Damping resistance $R_{t}($ Ohm $)$ & 100 & 100 \\
\hline Number of turns $N($ Turn $)$ & 38 & 38 \\
\hline
\end{tabular}

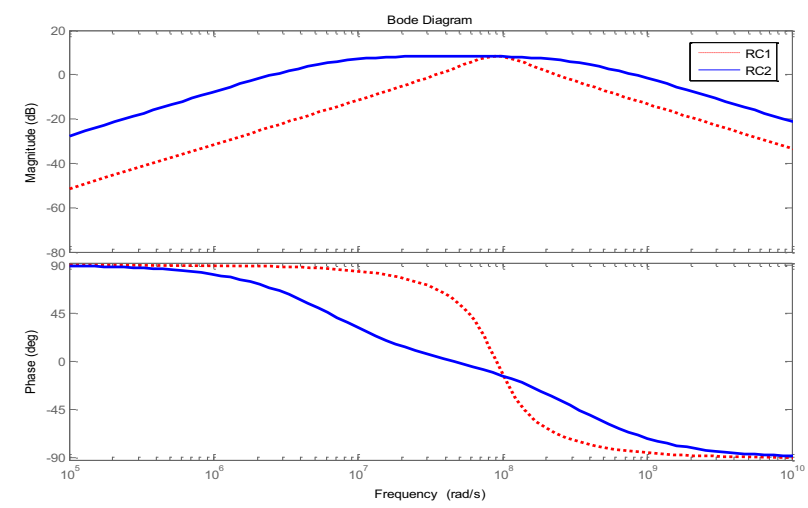

Figure 5. Frequency response of two coils.

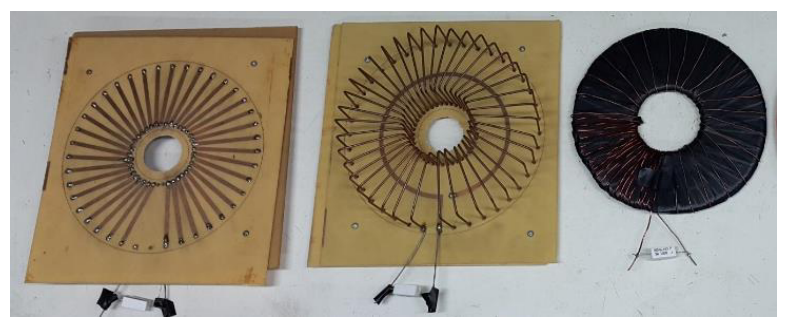

Figure 6. Photo of three coils.

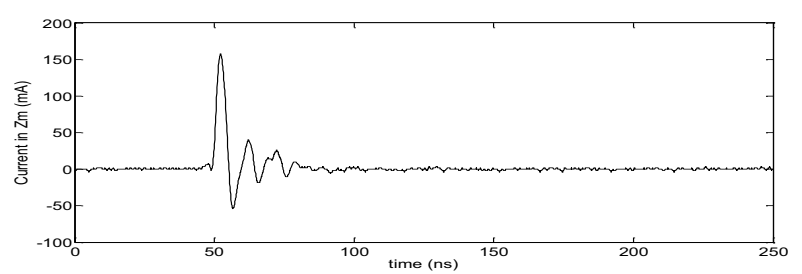

Figure 7. Current pulse.

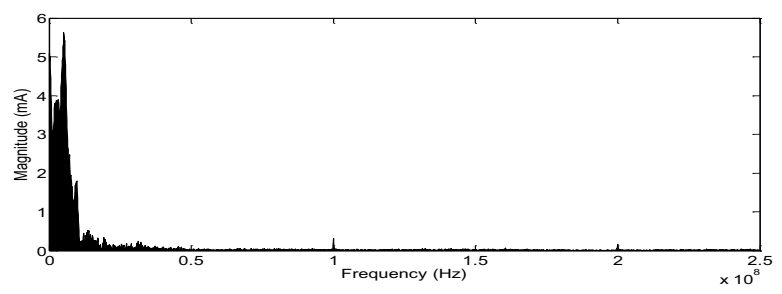

Figure 8. Spectrum of the current pulse.
The measured waveforms are shown in Fig. 9, 10, 11.The output signal from Combine Rogowski coil has higher amplitude than that of the other coils because of high mutual inductance and also its output doesn't oscillate which is the good characteristic of PCB Rogowski coil.

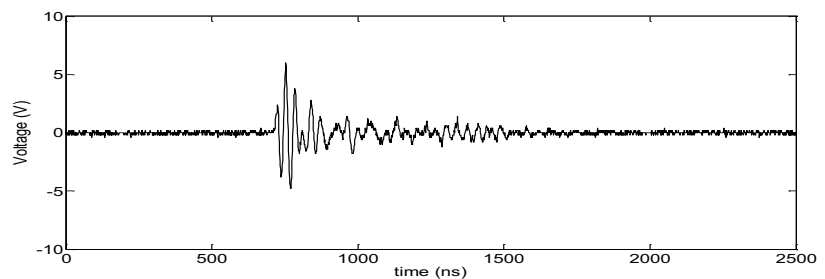

Figure 9. Pulse detected by RC1.

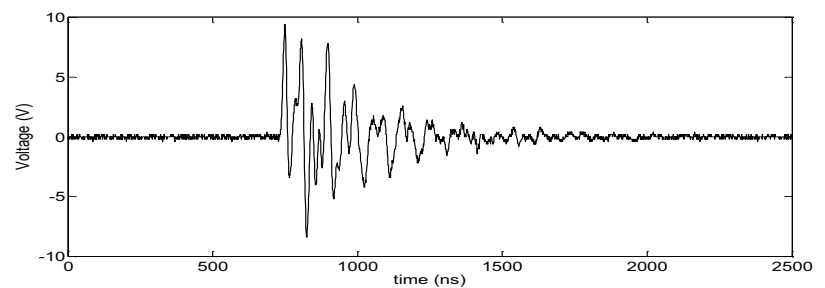

Figure 10. Pulse detected by RC2.

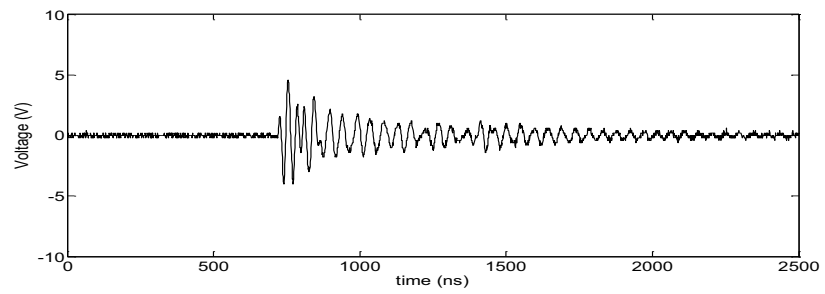

Figure 11. Pulse detected by non-uniform RC.

\section{Optimization}

Genetic algorithm is a method for finding an optimum values of problem based on a natural selection process which mimics biological evolution, when considering the problem in real life there are more than one objectives, optimizing for a particular objective may result to the unacceptable solution to the other objective. So that a multi-objective optimization problem was investigated, a set of solution where each set of solution satisfies the objectives at acceptable point without dominated by any other solution as call Pareto front [8].

For tuning Rogowski coil to be able to measure a small current, we must improve the mutual inductance by increasing the height of the core or number of turns. Nevertheless, increasing of these parameters will also improve inductance of coil which means the sensitivity of coil at high frequency might be diminished.

According to the equation (7), upper frequency limitation can be found

$f_{h}=\frac{\left(L+R R_{t} C\right)+\sqrt{\left(L+R R_{t} C\right)^{2}+4\left(R_{t} L C\right)\left(R+R_{t}\right)}}{4 \pi R_{t} L C}$

And the value of suitable damping resistance can be determined as [9]. 


$$
R_{t}=\frac{1}{2} \sqrt{\frac{L}{C}}
$$

From the equation (8), (9), the upper frequency limitation can be rewritten as

$$
f_{h}=\frac{\left(L+\frac{R}{2} \sqrt{L C}\right)+\sqrt{2 L^{2}++3 R L \sqrt{L C}+\frac{1}{4} R^{2} L C}}{2 \pi L \sqrt{L C}}
$$

Using Rogowski coil for measuring a very low current, but with a very high frequency such as PD signals have been very attracted moreattention. The challenge is the requirement of a Rogowski coil with high sensitivity and wide bandwidth that can cope up with the PD signals.

Objective functions for maximization are mutual inductance from (2) which directly related with sensitivity of Rogowski coil and upper frequency limitation from (10). These $R, L, C$ parameters are a function of geometric parameters $a, b, h, N$ and its constraints according to the inside diameter based design method (IDDM) [10] are shown in Table 2.

Table 2. Geometric constraints.

\begin{tabular}{|c|c|}
\hline \hline Parameters of coil & Constraints value \\
\hline Inner radius $a(m)$ & $0.035-0.05$ \\
\hline Outer radius $b(m)$ & $0.057-0.137$ \\
\hline Height of core $h(m)$ & $0.01-0.05$ \\
\hline Number of turns $N($ turns $)$ & $50-82$ \\
\hline
\end{tabular}

When analyze multi objective genetic algorithm tool in Matlab by setting population size of 100 groups, cross over fraction 0.7 and pareto fraction 0.1 then, we shall get a group of solution as shown in Table 3 and Fig. 12, shows Pareto front(Local pareto solution).

\begin{tabular}{|c|c|c|c|c|c|c|c|}
\hline \multirow[t]{2}{*}{$\overline{\text { No. }}$} & \multicolumn{5}{|c|}{ Parameters } & \multirow{2}{*}{$\begin{array}{c}\boldsymbol{M} \\
(\boldsymbol{\mu H})\end{array}$} & \multirow{2}{*}{$\begin{array}{c}\boldsymbol{f}_{\boldsymbol{h}} \\
(\boldsymbol{M H z})\end{array}$} \\
\hline & $\begin{array}{c}a \\
(\boldsymbol{m})\end{array}$ & $\begin{array}{c}b \\
(\boldsymbol{m})\end{array}$ & $\begin{array}{c}\boldsymbol{h} \\
(\boldsymbol{m})\end{array}$ & $\begin{array}{c}N \\
\text { (Turns) }\end{array}$ & $\begin{array}{c}R_{t} \\
(\mathbf{O h m})\end{array}$ & & \\
\hline 1 & 0.0477 & 0.1369 & 0.049 & 79 & 595.33 & 0.8226 & 7.204 \\
\hline 2 & 0.0499 & 0.0801 & 0.010 & 82 & 318.21 & 0.0818 & 37.70 \\
\hline 3 & 0.0498 & 0.0841 & 0.010 & 82 & 318.05 & 0.0912 & 34.02 \\
\hline 4 & 0.0477 & 0.1369 & 0.049 & 79 & 595.33 & 0.8226 & 7.204 \\
\hline 5 & 0.0446 & 0.0826 & 0.014 & 73 & 353.20 & 0.1331 & 28.76 \\
\hline 6 & 0.0467 & 0.0826 & 0.013 & 77 & 350.17 & 0.1146 & 30.92 \\
\hline 7 & 0.0443 & 0.0895 & 0.022 & 73 & 434.13 & 0.2312 & 20.24 \\
\hline 8 & 0.0474 & 0.0832 & 0.011 & 78 & 324.73 & 0.0992 & 32.99 \\
\hline 9 & 0.0464 & 0.1190 & 0.049 & 76 & 615.25 & 0.7068 & 8.856 \\
\hline 10 & 0.0438 & 0.0811 & 0.025 & 72 & 467.75 & 0.2226 & 22.63 \\
\hline
\end{tabular}

Table 3. Set of pareto optimal solutions.

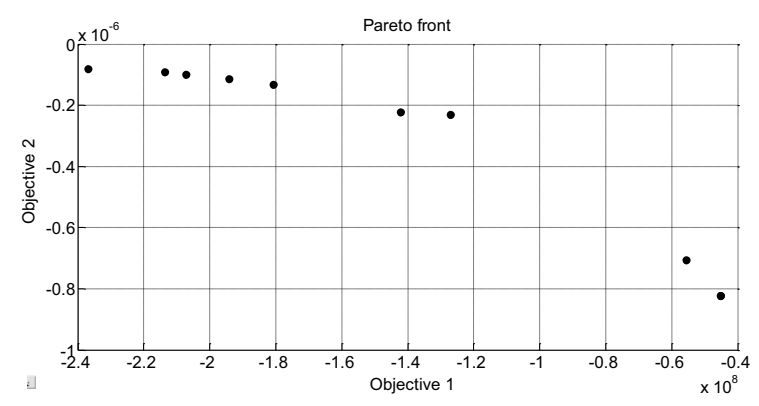

Figure 12. Pareto front.

\section{Conclusion}

The proposed Combined Rogowski coil has been constructed. There are many advantages on small signal detection due to low value of coil's capacitance that will improve the upper frequency limitation. Also, the coil has more cross-section area leads to high mutual inductance and uniform winding to diminish the oscillation from output signal. The optimization technique using multiobjective genetic algorithm is applied for improving the sensitivity and the bandwidth of coil in order to achieve the best optimum permissible value.

\section{References}

1. D. A. Ward, J.L.T. Exon, Engineering Science and Education Journal, Using Rogowski coils for transient current measurements, 2, pp. 105-113, (1993)

2. W. Chengyuan, ICMA,Design of Printed-Circuit Board Rogowski Coil for Highly Accurate Current Measurement, pp. 3801-3806, (2007)

3. Y. Zhang, Power System Technology Conference, The reliable design of PCB Rogowski Coil current transformer, pp. 1-4,(2006)

4. L. Kojovic, IEEE Computer Applications in Power, PCB Rogowski Coils Benefit Relay Protection, 15, pp. 50-53, (2002)

5. M. Argueso, Scientific Instruments, implementation of Rogowski coil for the measurement of partial discharges, 76, (2005)

6. M. Shafiq,Elsevier Measurement, Effect of geometrical parameters on high frequency performance of Rogowski coil for partial discharge measurements, 49, pp.126-137, (2014)

7. G. Robles, Instrumentation and Measurement Technology Conference Proceedings, Identification of parameters in a Rogowski coil used for the measurement of partial discharges, pp. 1-4, (2007)

8. K. Deb, Chichester Wiley, Multi-Objective Optimization Using Evolutionary Algorithms, (2001)

9. M. Shafiq, Sensors Journal IEEE, Parameters Identification and Modeling of High-Frequency Current Transducer for Partial Discharge Measurements, 13, pp. 1081-1091, (2012)

10. D. Porto, 3th IAS, Design of a new air-cored current transformer part analytical modeling and experimental validation, 1, (2004) 